\title{
ChemComm
}

Check for updates

Cite this: Chem. Commun., 2020, 56,7108

Received 9th April 2020,

Accepted 19th May 2020

DOI: $10.1039 / \mathrm{d} 0 \mathrm{cc} 02582 \mathrm{~h}$

rsc.li/chemcomm

\section{Cell-free protein synthesis in hydrogel materials $\dagger$}

\author{
Colette J. Whitfield, (D) a Alice M. Banks, (D) a Gema Dura, (D) a John Love, (D) ${ }^{b}$ \\ Jonathan E. Fieldsend, (D) ${ }^{c}$ Sarah A. Goodchild, ${ }^{d}$ David A. Fulton (D) ${ }^{a}$ and \\ Thomas P. Howard (D) *a
}

\begin{abstract}
We report a method for embedding cell-free protein synthesis reactions in macro-scale hydrogel materials without a free liquid phase. This paper focuses on methods of preparation for a variety of hydrogels and an investigation of the impact that the hydrogel material has on cell-free protein synthesis.
\end{abstract}

Cell-free protein synthesis (CFPS) uses cellular transcriptional and translational machinery to synthesise proteins outside the living cell. ${ }^{1}$ CFPS systems have shown potential as diagnostic tools in the detection of Zika and Ebola viruses, ${ }^{2,3}$ as well as for detection of metabolically important molecules such as hippuric acid and cocaine. ${ }^{4}$ CFPS is typically performed in liquid reactions in vitro, though there have been recent developments in the use of cell-free protein synthesis on paper supports and with microgels fabricated from DNA, ${ }^{5}$ agarose, ${ }^{6}$ clay, ${ }^{7}$ hyaluronan, ${ }^{8}$ polyacrylamide, ${ }^{9}$ fibrin and PEG-peptide. ${ }^{10}$ In each instance microgels are prepared and incubated within CFPS reaction mixtures, ${ }^{5,6,8,9}$ or hydrogel polymers form around an active liquid CFPS reaction. ${ }^{7,10}$ As such we cannot exclude the possibility that some aspects of the CFPS reaction (i.e. transcription or translation) occur in the reaction solution outside the gel and protein products diffuse into the gel. Alternatively, protein synthesis may occur before the gel has formed, producing a functional enzyme in liquid phase. Subsequent gel polymerisation may be the result of the enzyme product rather than active CFPS reactions.

Importantly, hydrogels have valuable properties in their own right. They act as pastes, ${ }^{11}$ lubricants ${ }^{12}$ and adhesives ${ }^{13}$ or in

\footnotetext{
${ }^{a}$ School of Natural and Environmental Sciences, Faculty of Science, Agriculture and Engineering, Newcastle University, Newcastle, UK.

E-mail: thomas.howard@newcastle.ac.uk

${ }^{b}$ Biosciences, College of Life and Environmental Sciences, University of Exeter, Exeter, UK

${ }^{c}$ Computer Science, College of Engineering, Mathematics and Physical Sciences, University of Exeter, UK

${ }^{d}$ Defence Science and Technology Laboratory, Porton Down, Wiltshire, UK

$\dagger$ Electronic supplementary information (ESI) available: Materials, methods, supplementary data and hydrogel characterisation. See DOI: 10.1039/d0cc02582h
}

the provision of structural support for regenerative cell growth. ${ }^{14}$ Considering the broad applications of hydrogels and the functionality of CFPS, we hypothesise that hydrogel matrices may act as a physical chassis for CFPS - allowing CFPS to move from a predominantly in vitro application into a physical, biologically activated material. If CFPS reactions are to be deployed in a material chassis, it is critical that the reactions occur within the material and without a separate liquid phase, and that we understand the impact hydrogels themselves may have on CFPS. Moreover, it is also important to explore how broadly suitable macro-scale hydrogels are as physical chassis for CFPS. Previous studies conducting CFPS in hydrogels typically use microgel systems ranging from 1 to $400 \mu \mathrm{m}$ in size. ${ }^{5-10}$ Here, we describe methods of embedding cell-free cellular components capable of performing protein synthesis in hydrogels (Scheme 1). Using these approaches, we demonstrate that CFPS can be performed throughout a range of hydrogel materials synthesised at macro-scale $(2-20 \mathrm{~mm})$ and in the absence of an external liquid phase. Moreover, the methods allow us to probe the physical effect that hydrogels can have on the performance of CFPS.

First, we established gene expression of mCherry in agarose gels using method (a) (Scheme 1 and Fig. 1a). Briefly, gels were prepared by the addition of molten $\left(60{ }^{\circ} \mathrm{C}\right) 3 \%$ agarose to cellfree reaction components in the appropriate cast. Gels were allowed to set for $30 \mathrm{~min}$ at room temperature before incubation at $37^{\circ} \mathrm{C}$. All reaction components were situated within the hydrogel polymer network; there was no external liquid observed in the system. We monitored the appearance of mCherry fluorescence over $16 \mathrm{~h}$ using spectroscopy and confirmed fluorescence within the gels using confocal microscopy. Our data indicated that mCherry fluorescence increased throughout the time period, and was detected and located evenly throughout the material (Fig. 1a, b and ESI, $\dagger$ Fig. S1). As the fluorescence increase was continuous, we conclude that $\mathrm{CF}$ components are stable in gels at $37{ }^{\circ} \mathrm{C}$ for $16 \mathrm{~h}$.

With an analytical system established, we next determined whether CFPS is compatible with a broad range of hydrogel 
(a)

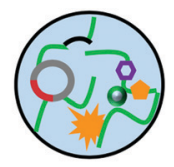

(b)

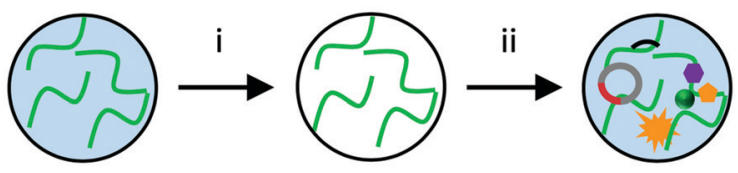

(c)

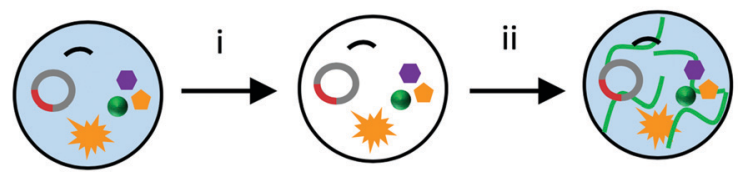

Key to components:

\section{$\checkmark$ Polymer \\ mCherry DNA}
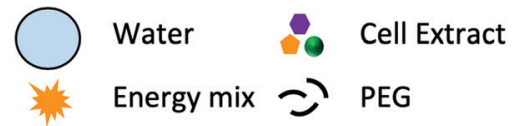

Scheme 1 Three preparation methods to embed cell-free reagents in macro-scale hydrogels: (a) CFPS reaction components and hydrogel polymers are combined immediately, (b) the hydrogel is prepared, freeze-dried and reconstituted using cell-free reaction components and (c), the cell-free reaction components are freeze-dried and reconstituted with a liquid hydrogel. For each method (i) denotes a freeze-drying stage and (ii) denotes a reconstitution stage.

materials. Three fabrication methods were used to integrate CFPS reaction components within the hydrogels in the absence of an external liquid phase (Scheme 1). For method (a), each component was prepared fresh, i.e. hydrogel components and freshly prepared cell-free components were added together and allowed to set before analysis. In some cases, cell-free components were added directly to solid polymer powders whereas others were added to concentrated liquid hydrogels. In method (b), the gel was prepared and allowed to set in the appropriate vessel, for example a 384-well plate or Petri dish, freeze-dried and reconstituted with freshly prepared cell-free reaction components. The gel was then allowed to re-form for $30 \mathrm{~min}$ before instigating further experimental procedures. The appropriate volume to reconstitute each hydrogel can be calculated from the \% reconstitution in Table S1, ESI. $\dagger$ In method (c), the CFPS reaction components were combined on ice, freeze-dried, and reconstituted with liquid hydrogel. Again, the gel was allowed to reform for $30 \mathrm{~min}$ prior to further work. Rheological analysis of each material was performed to confirm that all materials were gels (Fig. S2 and Table S2, ESI $\dagger$ ). To ascertain active CFPS in these gels, we expressed the mCherry reporter protein. We analysed expression in 12 gels prepared by each possible method with a variety of different structural and chemical characteristics, including entangled polymers, micellar aggregates and covalently crosslinked materials. A variety of polymer weight to volume $(\mathrm{w} / \mathrm{v})$ ratios were examined to determine the optimum concentration for fluorescent output, see Fig. S3 and Table S3, ESI. $\dagger$ For some hydrogels, all methods were appropriate, whilst for others, only one method was feasible and may have required additional steps, such as dialysis. We monitored (a)

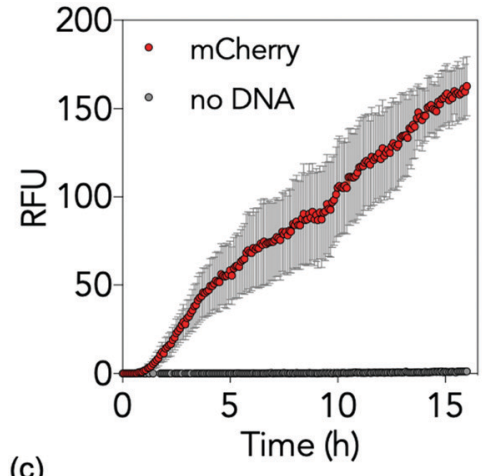

(b)

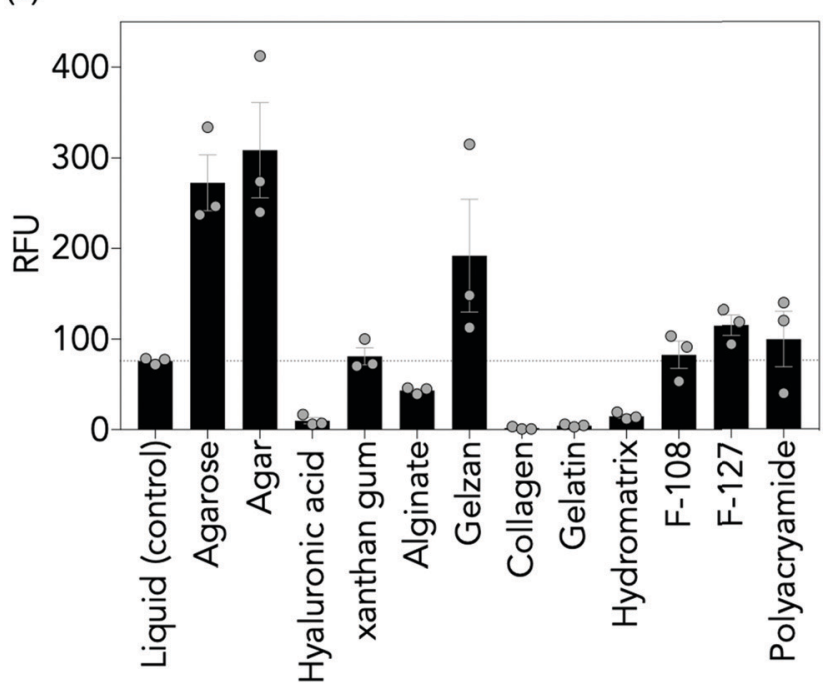

Fig. 1 CFPS in hydrogels. (a) Time course of cell-free mCherry expression in agarose in the presence and absence of mCherry coding DNA $(n=3$, error bars are standard error (SE) mean. Relative fluorescence units (RFU)). (b) Expression of mCherry is visible throughout the gel. Confocal microscopy of a central plane shows fluorescence of mCherry in agarose. Scale bar $=10 \mathrm{~mm}$. (c) CFPS is possible in a range of materials. RFU detected from a range of hydrogels containing cell-free reaction mixtures and mCherry template DNA. RFU is represented as the max RFU detected over a $16 \mathrm{~h}$ period. Hydrogel $\mathrm{w} / \mathrm{v} \%$ plotted; agarose: $1 \%$, agar: $1 \%$, hyaluronic acid: $5 \%$, xanthan gum: $0.5 \%$, alginate: $5 \%$, Gelzan ${ }^{\mathrm{TM}}$ : $2 \%$, collagen: $1 \%$, gelatin: $10 \%$, HydroMatrix ${ }^{\mathrm{TM}}$ : $1 \%$, F-108: $30 \%$, F-127: 30\%, polyacrylamide: $5 \%(n=3$, error bars are SE mean).

the fluorescence output for mCherry and compared the maximum fluorescence observed over a $16 \mathrm{~h}$ period. Two controls were employed: a positive control (CFPS of mCherry in liquid phase) and a negative control (CFPS reaction mixture lacking a DNA template to ensure all fluorescence detected could be attributed to mCherry protein synthesis, ESI, $\dagger$ Fig. S3). Overall, our data indicated that the ability to perform CFPS in hydrogels, though variable, is possible in different matrices (Fig. 1c, ESI, $\dagger$ Table S3 and Fig. S3). Of the 12 gels tested, six were polysaccharide-based hydrogels: agarose, agar, xanthan gum, hyaluronic acid, Gelzan ${ }^{\mathrm{TM}}$ and alginate. These gels feature extensive, non-covalent linkages between polymer chains, formed by hydrogen bonding and van der Waals forces (agarose, agar, xanthan and hyaluronic acid) or divalent ionic bridges $\left(\mathrm{Mg}^{2+}\right.$ for Gelzan ${ }^{\mathrm{TM}}$ and $\mathrm{Ca}^{2+}$ for alginate). For agarose, 
agar, xanthan, and Gelzan ${ }^{\mathrm{TM}}$ the fluorescence detected was equivalent to, or up to $400 \%$ greater than the fluorescence observed when CFPS was conducted in liquid phase. For alginate and hyaluronic acid in contrast, there was a reduction in fluorescence of between 13 and 57\% respectively compared to the control. Three amino acid-based hydrogels were also assessed: one protein hydrogel (collagen) and two peptide hydrogels (gelatin and HydroMatrix ${ }^{\mathrm{TM}}$ ). These gels are formed through hydrophobic interactions, $\pi-\pi$ stacking, hydrogen bonding and electrostatic interactions between polymers. ${ }^{15}$ For each of the three peptide-based hydrogels CFPS was observed above baseline but fluorescence signals were low, reaching between 6 and $43 \%$ of that observed in the positive control. Pluronic acid F-108 and F-127 are poloxamer gels where individual micelles aggregate to form a gel. For these gels, fluorescence was equivalent to, or up to, $120 \%$ of the liquid phase control. Finally, an examination of CFPS in a polyacrylamide gel, a covalently crosslinked network formed from the polymerisation of acrylamide and bis-acrylamide, revealed that CFPS-fluorescence was $110 \%$ of that observed in the liquid phase control reaction. CFPS is therefore possible in a range of hydrogel materials and, in some instances, performing the reaction in a hydrogel chassis results in an increase in the fluorescent output of the reaction. It follows that choice of hydrogel chassis for CFPS is not restrictive, and that a gel can be selected that is appropriate to the proposed end-use.

To confirm that gene expression occurs within the material, confocal microscopy was used to observe mCherry fluorescence throughout the gels (Fig. 2 and Fig. S4, ESI $\dagger$ ). Gels demonstrating equivalent or increased fluorescence compared to control reactions (agarose, agar and xanthan gels) displayed homogenous mCherry-fluorescence throughout the gel. Others, in which cell-free production of mCherry was detected but reduced (e.g. hyaluronic acid) demonstrated heterogeneous patterns of fluorescence. Scanning Electron Microscopy (SEM) (Fig. 2) of a selection of gels suggests that large, open pores correspond with homogenous patterns of gene expression in comparison to those with smaller, or more irregular shaped pores. Additionally, the presence of homogenous and regular pore sizes also corresponds with higher fluorescence outputs. We therefore hypothesised that the ability of reagents to diffuse through the material would significantly impact on CFPS function. To test this, we examined the diffusion of fluorescein (a small molecule fluorophore) at different agarose $\mathrm{w} / \mathrm{v}$ ratios. We observed that an increase in pore size correlated with an increase in observed diffusion (Fig. S5, ESI $\dagger$ ) while increasing $\mathrm{w} / \mathrm{v}$ ratio and decreasing the pore size correlated with a decrease in diffusion ability. This data along with the confocal and SEM observations support the view that gels with larger, more open pores allow greater diffusion and therefore a higher level of protein synthesis.

Macromolecular crowding is an established mechanism that positively influences CFPS reactions. ${ }^{16}$ Macromolecular molecules (commonly polyethylene glycol (PEG) in CFPS reactions) are present at a high concentration resulting in an increase of local effective concentration for the reaction's substrates. Given

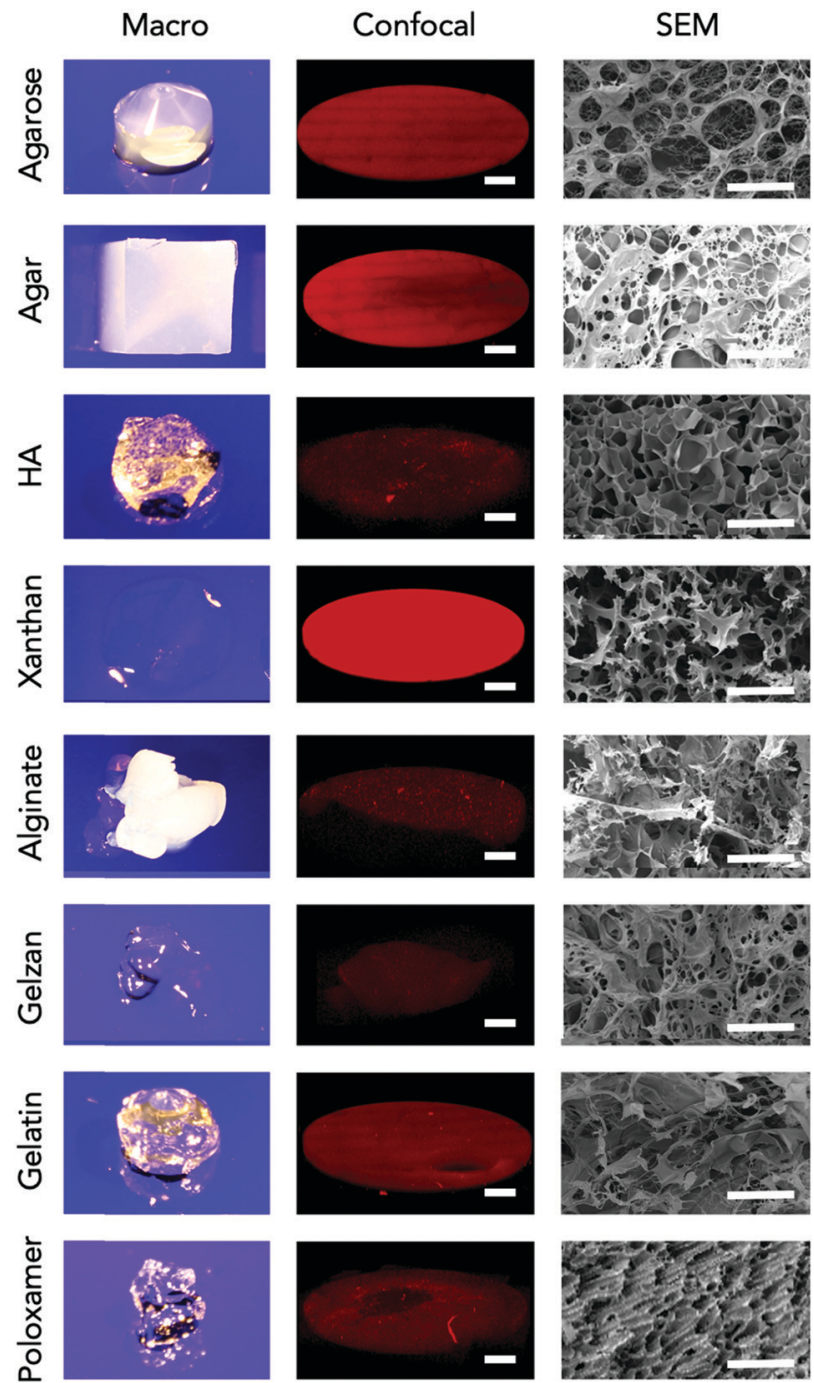

Fig. 2 The hydrogels used in this study exhibit a range of macro- and micro-scale structures. Hydrogel images of the macrostructure (left), confocal fluorescence microscopy of a central plane of the hydrogels after $4 \mathrm{~h}$ of incubation at $37{ }^{\circ} \mathrm{C}$ with the CF components and mCherry coding DNA (middle, scale bar $=1 \mathrm{~mm}$. Excitation and emission parameters were constant across all hydrogels) and scanning electron microscopy (SEM) of each material (right, scale bar $=100 \mu \mathrm{m})$.

that diffusion is not the sole factor influencing CFPS output, we hypothesised that the hydrogel network may also act as a macromolecular crowder. To test this, we assayed CFPS in agarose gels without the crowding agent PEG and with PEG at half the standard concentration (1\%). Reactions in the absence of agarose and lacking PEG demonstrated reductions in CFPS dependent fluorescence of $72 \%$ while those at half standard PEG concentrations demonstrated reductions of $42 \%$ (Fig. 3 inset). The data indicated that $1 \%$ agarose gels without PEG maintained fluorescence values equivalent to those observed in standard liquid phase reactions with $2 \%$ PEG. Moreover, reductions in CFPS output observed when PEG concentrations are reduced by half are more than recovered by performing the reactions in 1 or $2 \%$ agarose gels (Fig. 3 ). This data supports the 


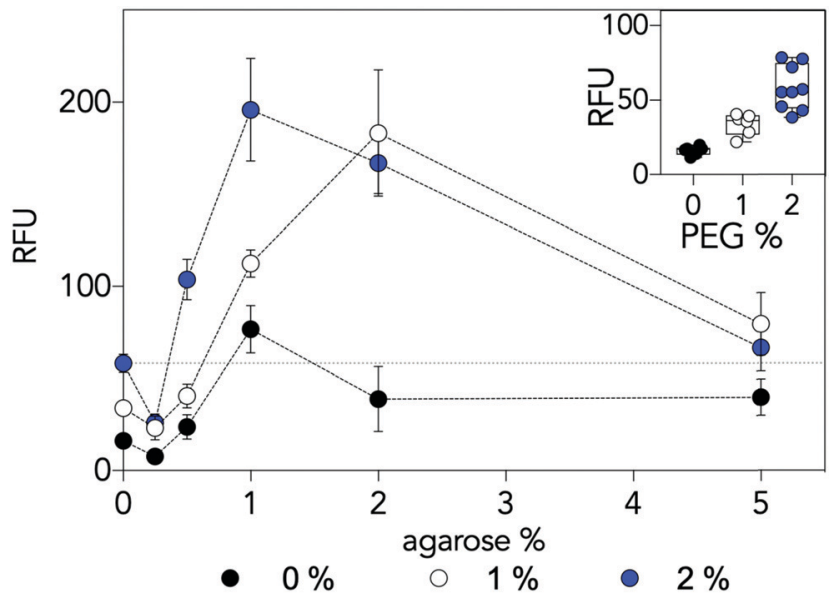

Fig. 3 Cell-free mCherry protein synthesis at increasing concentrations of agarose in the absence $(0 \%)$, or in the presence of $1 \%$ or $2 \%$ polyethylene glycol (PEG) ( $n \geq 6$, error bars are SE mean). The horizontal dashed line indicates performance of the standard liquid phase reaction in the presence of $2 \%$ PEG. The insert graph demonstrates the effect of crowding on standard liquid phase CFPS.

hypothesis that the hydrogel matrix acts as a structural crowding agent as well as a physical support.

Here, we have demonstrated the fabrication and function of CFPS reactions in a range of hydrogel materials in the absence of an external liquid phase. CFPS reactions were possible inside polysaccharide, proteinaceous, micellar and covalently crosslinked hydrogels. Moreover, we have shown internal gel diffusion and the ability of gels to act as a structural molecular crowding agent impacts CFPS reaction efficacy. Our data supports the view that the range of hydrogel polymers compatible with CFPS is broad, not narrow, meaning that the selection of hydrogel can be appropriate to its desired use. Importantly, though we see an apparent benefit in increased protein production in some gels, the broad application across a range of gels means that even with decreases in CFPS performance, if the gel provides a functional or structural benefit to the designer (for example biocompatibility or adhesive properties) then there may be a permissible trade-off. Some loss of CFPS capacity may be tolerated for a gain of function that a particular gel may offer. However, while it is likely that the choice of reporter can be expanded to other fluorescent and chromophore reporter proteins, the key to broadening the applications of this approach will be to express and detect non-reporter proteins, and to express multiple proteins simultaneously, whose combined action enhances hydrogel functionality. Challenges in expressing non-reporter proteins will likely lie in the ability to assay functional protein synthesised within a gel matrix. Despite these difficulties, we propose that implementing cell-free gene expression in hydrogel materials will allow their deployment as physical biologically activated standalone materials.

The authors greatly acknowledge the support of the Engineering and Physical Sciences Research Council - Defence Science and Technology Laboratories award EP/N026683/1 (CJW, AMB, JL, JEF, DAF, TPH) and the Biotechnology and Biological Sciences Research Council award BB/M018318/1 (GD, DAF). Data supporting this publication is openly available through a CC0 1.0 Universal (CC0 1.0) Public Domain Dedication at: https://doi.org/10.25405/data.ncl.12349352.

\section{Conflicts of interest}

There are no conflicts to declare.

\section{Notes and references}

1 C. E. Hodgman and M. C. Jewett, Metab. Eng., 2012, 14, 261.

2 K. Pardee, A. A. Green, M. K. Takahashi, D. Braff, G. Lambert, J. W. Lee, T. Ferrante, D. Ma, N. Donghia, M. Fan, N. M. Daringer, I. Bosch, D. M. Dudley, D. H. O'Connor, L. Gehrke and J. J. Collins, Cell, 2016, 165, 1255.

3 K. Pardee, A. A. Green, T. Ferrante, D. E. Cameron, A. D. Keyser, P. Yin and J. J. Collins, Cell, 2014, 159, 940.

4 P. L. Voyvodic, A. Pandi, M. Koch, I. Conejero, E. Valjent, P. Courtet, E. Renard, J.-L. Faulon and J. Bonnet, Nat. Commun., 2019, 10, 1697.

5 (a) J. S. Kahn, R. C. H. Ruiz, S. Sureka, S. Peng, T. L. Derrien, D. An and D. Luo, Biomacromolecules, 2016, 17, 2019; (b) N. Park, S. H. Um, H. Funabashi, J. Xu and D. Luo, Nat. Mater., 2009, 8, 432.

6 J. Y. Byun, K. H. Lee, K. Y. Lee, M. G. Kim and D. M. Kim, Lab Chip, 2013, 13, 886.

7 D. Yang, S. Peng, M. R. Hartman, T. Gupton-Campolongo, E. J. Rice, A. K. Chang, Z. Gu, G. Q. Lu and D. Luo, Sci. Rep., 2013, 3, 3165.

8 T. Heida, T. Köhler, A. Kaufmann, M. J. Männel and J. Thiele, ChemSystemsChem, 2020, 2, e1900058.

9 X. Zhou, H. Wu, M. Cui, S. N. Lai and B. Zheng, Chem. Sci., 2018, 9, 4275 .

10 A. Huang, P. Q. Nguyen, J. C. Stark, M. K. Takahashi, N. Donghia, T. Ferrante, A. J. Dy, K. J. Hsu, R. S. Dubner, K. Pardee, M. C. Jewett and J. J. Collins, Sci. Adv., 2018, 4, 5105.

11 F. Yoshii, L. Zhao, R. A. Wach, N. Nagasawa, H. Mitomo and T. Kume, Nucl. Instrum. Methods Phys. Res., Sect. B, 2003, 208, 320.

12 M. E. Freeman, M. J. Furey, B. J. Love and J. M. Hampton, Wear, 2000, 241, 129.

13 C. E. Brubaker and P. B. Messersmith, Biomacromolecules, 2011, 12, 4326.

14 A. C. Jen, M. C. Wake and A. G. Mikos, Biotechnol. Bioeng., 1996, 50, 357.

15 A. Dasgupta, J. H. Mondal and D. Das, RSC Adv., 2013, 3, 9117.

16 (a) B. R. Fritz, O. K. Jamil and M. C. Jewett, Nucleic Acids Res., 2015, 43, 4774; (b) C. Tan, S. Saurabh, M. P. Bruchez, R. Schwartz and P. Leduc, Nat. Nanotechnol., 2013, 8, 602. 